\title{
The use of generative design for the architectural solutions synthesis in the typical construction of residential buildings
}

\author{
Nikita Smorzhenkov and Elena Ignatova* \\ Moscow State University of Civil Engineering (MGSU) National Research University, 129337, \\ Moscow, Russia
}

\begin{abstract}
The study deals with the application of generative design technology in solving architectural and construction problems. Generative design is a powerful tool for creating design choices. It is necessary to formalize the generation process, set the necessary parameters, algorithms and restrictions. The purpose of the study is to determine the possibilities of the generative design complex application in the typical construction of residential buildings. It is proposed to use the structure, floor, and section of the building as the structural modules for generating variants of buildings. In turn, module variants can be created based on generative design. It is concluded that it is possible to create a whole system of structural and parametric synthesis of architectural solutions.
\end{abstract}

\section{Introduction}

Relevance: Digital technologies are actively developing and every year they are increasingly being introduced into all spheres of human life, rebuilding and optimizing familiar processes. Design is the most important type of activity in which a person is increasingly assisted by computing systems and information technology. So, for example, the emergence of computers and CAD made it possible to translate the design process into a digital space, and building information modeling technologies made it possible to collect all information about the structure, linking it in the form of a single digital model. However, only the design tools and the principles of organizing and storing the architectural project have evolved qualitatively, the design process itself, in its creative basis, has not changed dramatically: the architect independently determines almost all the designed object's parameters and consistently forms its geometry, realizing his ideas on the canvas, which is now integrated into the interface of a computer program [1]. Rebuilding the process of architectural design became possible only with the advent of a fundamentally new technology - generative (algorithmic) design. The technology has provided an opportunity to involve computer programs directly in the creative process of architects and designers, to speed up the design process and improve the quality of its results.

Purpose of work is to explore the prospects for the introduction of generative design technology in construction industry and determine the possibilities of its complex application

\footnotetext{
*Corresponding author: ignatova@mgsu.ru
} 
in the typical construction of residential buildings.

\section{Materials and methods}

In the course of this study, an analysis of materials from the scientific publications, developments and data from Russian and foreign information sources on the topic of generative design was carried out. A hypothesis about the prospects of using generative design for the synthesis of architectural solutions in the typical construction of residential buildings has been put forward.

Generative (algorithmic) design is a method of designing and designing a digital or physical product based on the parametric and generative models' generation [2] according to a predetermined set of mathematical algorithms. With this approach, the role of a person is to create the systems of scripts and algorithms that automatically generate a variety of projects based on the analysis and processing of input information. Such information is a set of parameters that the generated object must correspond to. Due to the computer ability to quickly process large amounts of data, designers and designers can vary the input information, and almost instantly create new variations of the project, until the result is the best match the vision of the author [4]. In the case when a designer needs to evaluate the effectiveness of options in terms of individual quantitative characteristics of an object, a computer can also help him in this. For such purposes, generative design uses algorithms for finding optimal solutions, which allow the system not only to independently generate many variants of an object, but also to compare them with each other and select the best modifications according to specified criteria and constraints. Most often neural networks and evolutionary algorithms are used for this in generative design systems, which are based on mathematical models of the mechanisms of natural evolution.

Thus, it is possible to identify the common stages when working with a generative design system, characteristic of all areas:

1. Problem statement - defining the generative algorithm goals.

2. Setting parameters (conditions) - defining the characteristics that should

match generated solutions.

3. Generation is the process of creating and visualizing solutions based on input parameters as a result of an algorithm.

4. Analysis and selection of options - the generated solution evaluation, and in case of dissatisfaction - restarting the algorithm with new parameters [3].

The main advantages of using algorithmic design include a significant reduction in the time for translating creative ideas into projects, an increase in the quality and efficiency of the resulting projects, the implementation of multivariate design solutions, as well as a reduction in possible errors by reducing the influence of the human factor [4].

Despite the fact that today there are areas in which generative design is applied and developed more actively, the approach itself is not limited to any particular area of application. Today, generative design is most widely used in the graphic and web design industries, as well as in industrial design. Relatively recently, generative technologies have begun to be introduced into the construction industry and have already become one of the most promising areas for the development of modern building information modeling systems. The most popular tools for creating generative algorithms in the field of architectural design are visual programming editors such as Dynamo and Rhinoceros 3D Grasshopper, working in conjunction with BIM systems Autodesk Revit and Graphisoft Archicad, respectively.

Unfortunately, due to the complexity of use, the lack of proven techniques, in reality generative design in the field of architecture is not yet used so extensively. Today generative architecture is represented by the individual unique objects, such as the Heydar Aliyev Center in Azerbaijan, which has the most complex curvilinear shape of facades, and the Voxman 
Music School at the University of Iowa, in which a ceiling shape with optimal acoustic characteristics was developed using generative design methods (Fig. 1) [5].

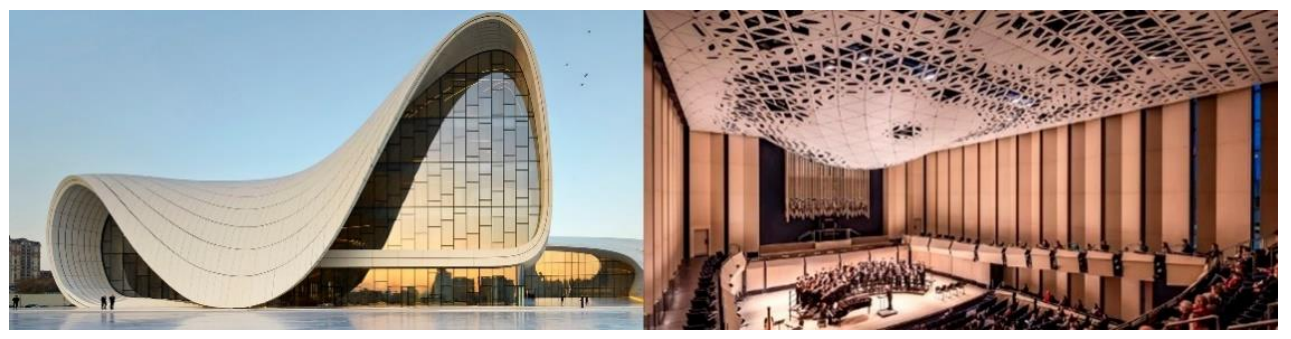

Fig. 1. Real projects created by generative design: the Heydar Aliyev Center and the ceiling of the Voxman music school.

Generative design tools are very flexible in use, and in addition to creating complex curvilinear structures, generative design is already used to solve a wide range of local optimization problems, such as the generation of building blocks, apartment design, interior furnishing, zoning of office premises, the problem of the optimal location of a building on a construction site. calculation of the best insolation, energy efficiency, and so on. The techniques for solving such problems are already being actively developed and are gradually beginning to be implemented in individual construction firms, allowing designers to save many hours by automating routine work and the ability to quickly generate and analyze a huge number of options. However, the development of generative design systems and information technologies in the future will make it possible to solve a whole range of design problems within one system and to carry out in the shortest possible time the generation of full-fledged building projects, that is, their automatic structural-parametric synthesis.

The concept of structural-parametric synthesis is associated with system analysis and denotes a process in which the structure of a system object is determined and the values of its constituent elements' parameters, methods of their connection and interaction are found so that the conditions of the synthesis task are satisfied [6].

In structural-parametric synthesis:

1) the structure of the model is unknown in advance and the model is generated automatically;

2) both the structure and the parameters change, that is, the search is carried out in the space of structures and parameters;

3) the number of parameters is unknown in advance and can be determined only after the structure has been defined.

Since a building is a system object, it has its own structure and parameters, the process of structural-parametric synthesis is applicable to architectural design. The architect's tools for implementing this process can be generative design algorithms. In this case, the tasks of the architect include drawing up the principles of the algorithms, the rules for the architectural models' formation and monitoring the system results. The algorithm automatically selects the structure of the building object and calculates the optimal parameters, synthesizing an architectural model that meets the conditions specified by the architect. There are already some promising developments in the field of generative architecture that have been able to solve the problem of synthesizing architectural solutions for a building object.

For example, the student of University of Nebraska Nate Holland developed a generative design system to automatically synthesize a multi-story building model using the Galapagos evolutionary search plug-in (Figure 2). In the process, its algorithm, developed by Grasshopper, selects the optimal building area, optimizes the upper and lower levels of the building, and also selects the optimal building shape, taking into account insolation. In 
addition, the author added to his system an algorithm for generating the optimal placement of apartments with the required number of rooms and area, capable of dividing an apartment into living rooms, rooms and bathrooms. As a result, the system generated many possible solutions, which took much more time and effort during traditional design [7].

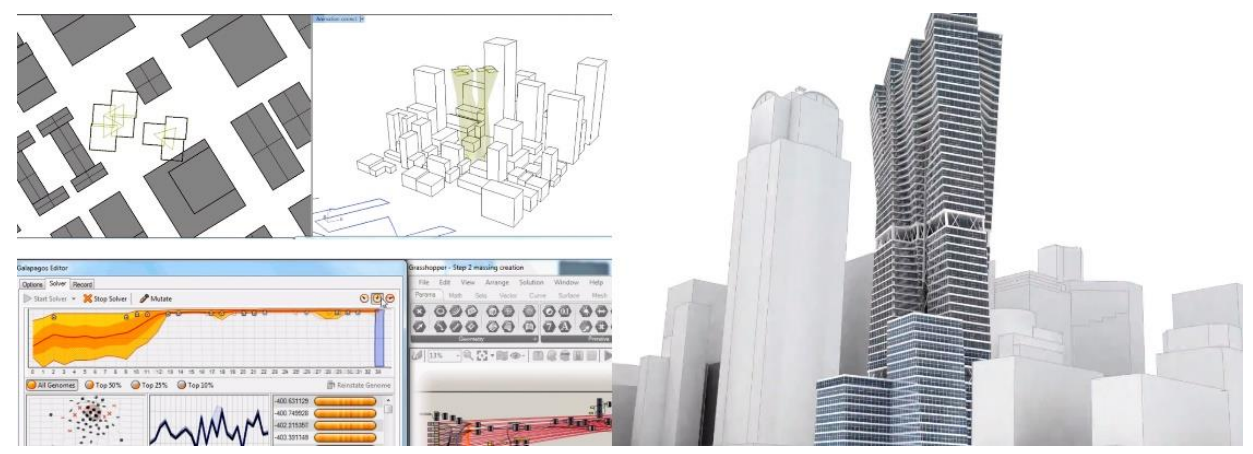

Fig. 2. A generative design system for the automatic synthesis of a multi-story building model, developed by Nate Holland.

An interesting development is described in the article [8]. The generative design system is able to synthesize a full-fledged information model of a one-story house in several stages, from the arrangement of primitive modules representing individual rooms that form the shape of a house, to the generation of a finished draft design of a house with internal and external walls, a pitched roof, windows and doors. The user of such a system only needs to indicate the number and types of premises in the future house, as well as the number of the cadastral construction site. The system takes into account many factors, such as climatic conditions, terrain, direction of the sun and the location of the road. According to the author's description, the process of executing the algorithm from launch to creating a finished project takes only 10 minutes [8].
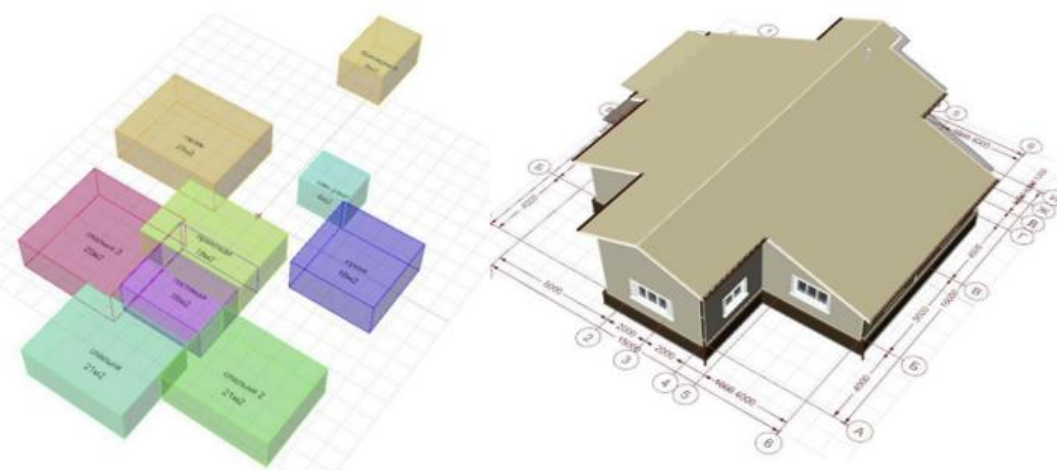

Fig. 3. Generative design system for low-rise residential buildings.

It is also worth noting the significant progress in the field of automatic building generation, which has been achieved by developers in the entertainment industry, creating applications to help video game developers, animators and special effects artists in cinema. Matt Estela, an artist at Side Effects Software, has developed a Building Generator plug-in for Autodesk 3ds Max using the Houdini graphics package with a built-in visual programming environment (Figure 4). As the name suggests, this versatile generative design system is able to generate buildings of any configuration with the ability to set the shape, number of storeys, and dimensions of the building and customize its individual elements, 
such as walls, windows, doors, exterior and interior decor, interior lighting, balconies, stairs, firefighters' stairs, columns, roof, attic and so on. In addition, it is possible to load a bunch of particular elements into the model and place them in the building as separate modules. The tool also makes it possible to set which parameters are assigned by the user and which will be chosen randomly during the generation process. And based on the building created in the plugin, it is possible to generate many similar structures [9].

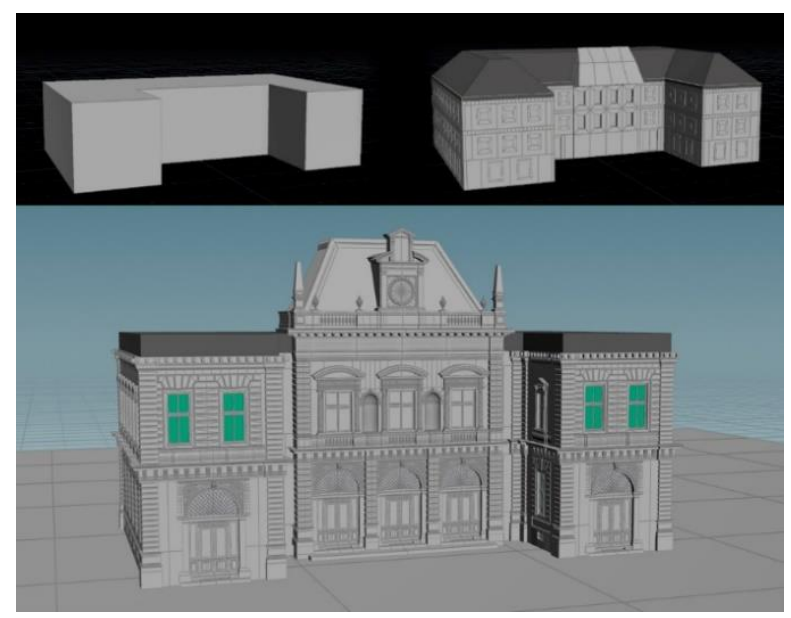

Fig. 4. Building Generator is a plugin for Autodesk 3ds Max.

Such generative design systems, developed using the Houdini graphics package, significantly simplify and speed up the work of designers involved in filling virtual worlds for video games, films and cartoons. "Building Generator" is not intended for use in the construction industry, therefore, it does not use either BIM technologies or optimization algorithms, because the only important criterion for the user of such a system is the external qualities of the generated model. However, such a plug-in can still act as a stimulator of the architect's creative process and provide assistance at an early stage of architectural design, when assistance is needed in defining the future building from the concept [10]. In addition, this plugin can serve as a guideline for how generative design systems should be arranged in the future.

The advantages and prospects of using generative design technology in construction were discussed in articles [11-14]. Among other things, the use of this technology in conjunction with building information modeling technology and additive construction production was discussed. The presented developments demonstrate the wide possibilities of using generative design algorithms for the automated creation of architectural solutions and solving a set of optimization problems.

\section{Results}

As a result of the study, the main advantages of generative design for the architectural solutions' synthesis were highlighted:

- $\quad$ design time reduction;

- $\quad$ improving the design quality and accuracy;

- $\quad$ increasing the efficiency of projects by selecting the optimal parameters;

- multivariance of design solutions;

- $\quad$ automatic discarding of invalid options. 
Typical construction of residential buildings can become one of the most promising areas for the generative design application. This is due to the fact that in standard construction, low terms and cost of design, quality and optimality of design solutions, as well as multivariate construction are of particular importance [15].

Modern buildings of typical construction, as a rule, have a sectional structure, which allows a modular approach to their design. With this approach, the building can be viewed as a complex system consisting of separate structural modulessubsystems, which in turn also consist of smaller subsystems. In typical residential buildings of a sectional type, modules can be sections, floors, apartments, and individual structural elements. All of these modules can be viewed as separate information models that form a hierarchical structure. An example of such a structure for a typical residential apartment building is shown in the diagram (Fig. 5). The number of modules in such a structure depends on the complexity of the building, as well as on the requirements of multivariance - the more modules, the more options for the building shape can be obtained.

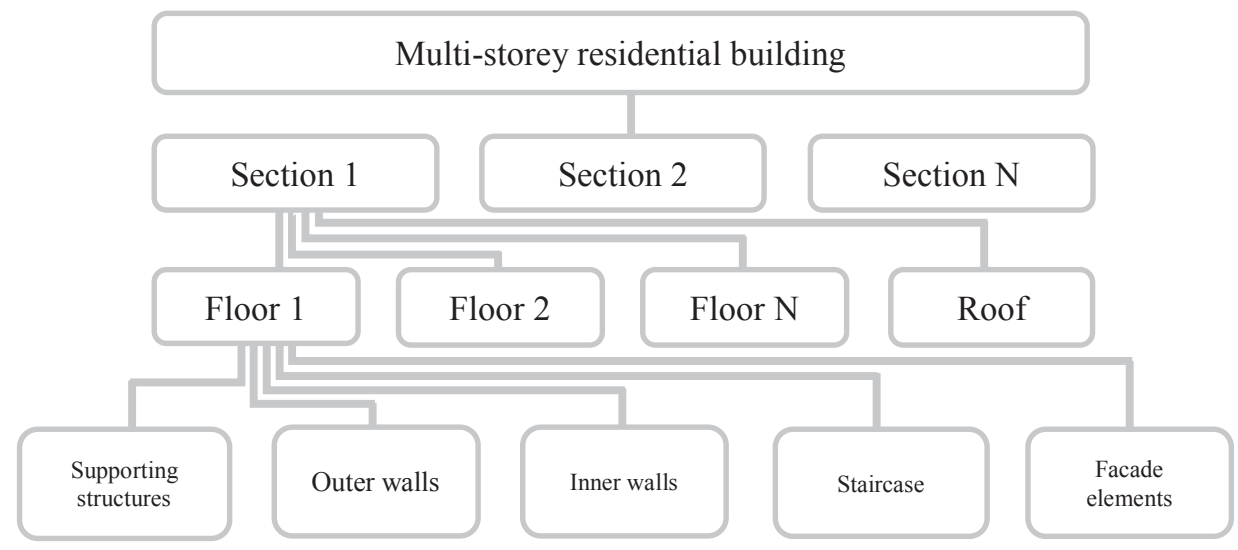

Fig. 5. Hierarchical structure of a multi-storey residential building of sectional type.

The following is an approximate algorithm and a description of the system's operation for the synthesis of architectural solutions for such a project of a residential building. This algorithm can be conditionally divided into several levels of design with the main sequential subtasks' implementation.

At the first stage generative design is done at the floor level. At this level, modules of typical floors are generated for various required types of building sections (ordinary, end, corner, etc.): the building area is determined for each separate section and the floor space is distributed into individual rooms. This process can be carried out in two ways: when the shape of the floor is determined by the rooms included in it (Fig. 7 a), or when the shape of the floor is first determined, which is then automatically subdivided into separate rooms using the apartment graphic methods (Fig.7 b). 


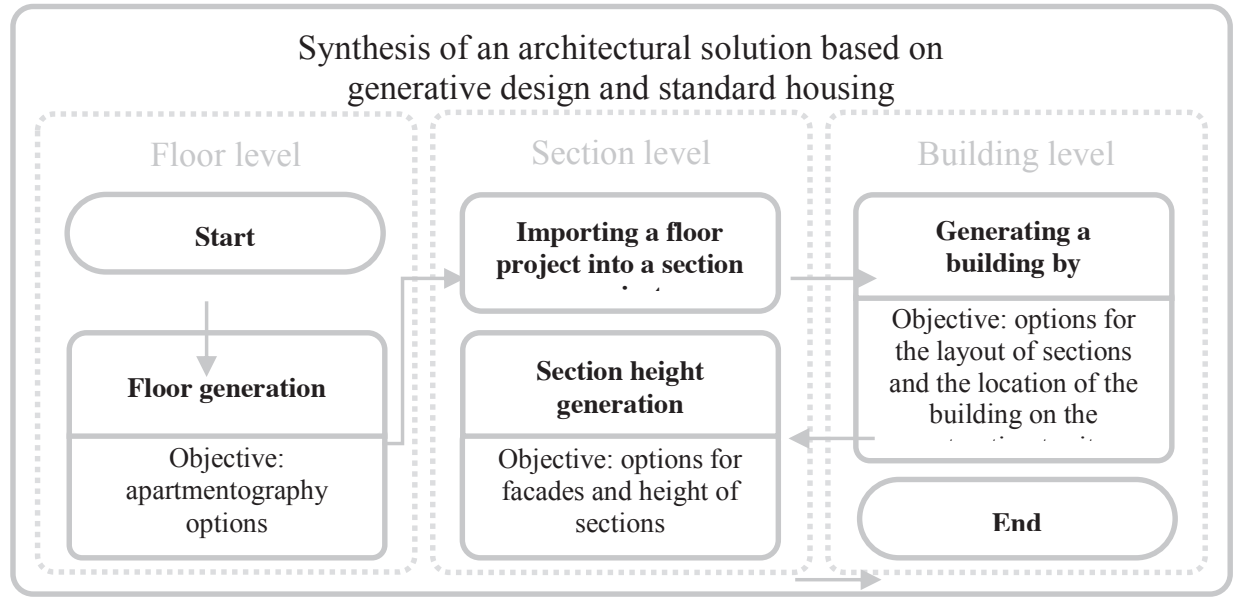

Fig. 6. An approximate algorithm of the system for the synthesis of architectural solutions for a typical project of a residential building.
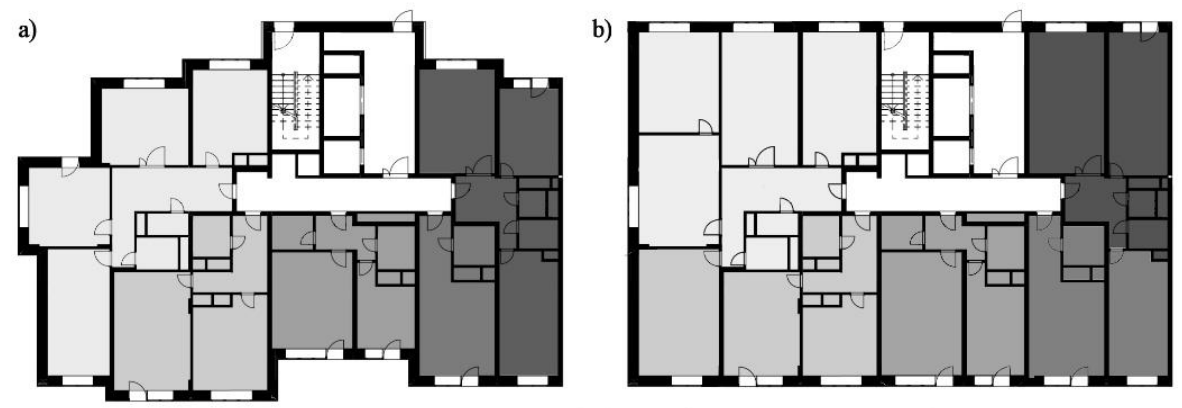

Fig. 7. Variants of section floor synthesis: a) shape based on rooms b) rooms based on shape

For both options, information is needed about the staircase, the number of apartments on the floor, the number and types and sizes of typical rooms in apartments and the rules for their joining, and other information necessary for correct generation. Minimization of the floor outer perimeter will act as an objective function in the process of allocating premises. It is also necessary to take into account information about the section type for which a typical floor is generated, since it determines not only the geometric shape of the section, but also the number of external walls for joining with adjacent sections (one for the closing section, two for all other types). It is also a limitation that the docking walls must be the same length for each type of section. Based on the generated floor plans, walls are generated, which, according to the specified rules, are supplied with door and window openings. Additional facade elements, such as balconies or loggias, are joined to the walls. After the completion of the generation of typical floors, on their basis, according to the specified rules, additional variations are generated for the first and last floors of the section.

In the second stage generative design is done at the section level. After receiving the typical floors of each type of sections, the received floor projects are loaded into the projects of the corresponding sections. At this stage, we do not care about the number of storeys in the sections, since it will be calculated later. Only the building spot that each section forms is important.

In the third stage generative design is done at the building level. At this stage in the building design, the building, formed by a sequence of joining projects of sections of different 
types, is assembled. At this stage, it is necessary to set the limits on the maximum and minimum number of sections in the building, as well as the rules for joining sections of different types with each other. The optimization tasks at this stage can be the tasks of finding the optimal building spot, as well as the location of the building, according to the relief, relative to the existing building and road system. To do this, the system needs information about the construction site and the existing infrastructure.

At the fourth stage generative design is done at the section level. After determining the sectional structure of the building and its location on the construction site, it is necessary to determine the number of storeys in each of its constituent sections. At this stage, it is necessary to set restrictions on the maximum and minimum number of floors in a section, as well as to define the rules for joining floors with each other. The number of storeys is determined by solving the optimization problem of calculating insolation in apartments, which will require entering information about the existing development and the orientation of the building relative to the cardinal points.

\section{Conclusion}

Generative design is one of the most promising directions in architectural design development, which has many significant advantages. The complexity of creation and use is a serious drawback of generative design systems and, so far, limits their implementation and widespread use in the construction industry.

The use of generative design can receive new development in certain areas of construction, such as standard and modular construction of multi-storey residential buildings. The synthesis of architectural solutions in the typical design of residential buildings can be greatly simplified, due to the fact that the architectural model of a building is generated not from individual elements, such as walls and ceilings, but is assembled from ready-made repeating volume-planning modules, which must be correctly and optimally placed in space of the project relative to each other.

The modules themselves can also be pre-generated to achieve the most optimal performance. The modular structure of the building allows not only to reuse the modules, but also allows you to replace some modules with analogs, change the order of arrangement and the number of modules.

The structural-parametric approach allows the solution of individual problems of variant design to be combined into a common system for the synthesis of architectural solutions of buildings.

By changing the structure of the building, you can vary the types, order and number of sections, select the number of floors in sections and choose options for finishing the facade or the layout of apartments on the floor. Due to this, a complex multivariate design is provided, which allows, on the basis of one modular structure, to synthesize many typical buildings that differ in volume and planning structure and have a unique appearance.

\section{References}

1. Don State Technical University, Computational architecture. https://donstu.ru/news/intervyu/vychislitelnaya-arkhitektura/ (access date: 01.05.2021).

2. Gip \& Gap Builders Engineering Club, Algorithmic design. http://gipgap.ru/obuchenie/povyshenie-kvalifikacii/algoritmicheskoe-proektirovaniemoskva/ (access date: 05.05.2021).

3. V.D. Slizh, V.B. Salnikov, Advantages of generative design, Ural TIM readings. Information Modeling Technologies for Buildings and Territories: Materials of the 
Scientific and Practical All-Russian Conference (Yekaterinburg: LLC "Agraph Typography"), Yekaterinburg, November 5-6, 23-27 (2020)

4. V.A. Malashenkova, A.Yu. Chernitskaya, Generative design is a revolutionary design method (In the collection: student of the year 2020. collection of articles from the International Research Competition. Petrozavodsk, 2020)

5. Autodesk Redshift, Generative Design in Architecture and Construction Will Pave the Way to Productivity. https://redshift.autodesk.com/generative-design-architecture/ (access date: 05.05.2021).

6. V.A. Volkov, S.M. Chudinov, Scientific Gazette. History Series. Political science. Economy. Informatics 19 (138) 24/1, 153-157 (2012)

7. M.I. Bzhakhov, M.M. Efimova, A.V. Zhurtov, Engineering Journal of Don 2 (49), 166 (2018)

8. D.O. Fedchun, Bulletin of the Engineering School of the Far Eastern Federal University 3 (36), 171-183 (2018)

9. Side FX, Building Generator. https://www.sidefx.com/tutorials/building-generator/ (access date: 05.05.2021).

10. X. Marsault, Eco-generative Design for Early Stages of Architecture (Architecture and Computer Science Set, London, 2018)

11. E.V. Ignatova, V.P. Predeina, Construction and architecture 9 (1) (30), (2021)

12. A.B. Parkhimovich, A.V. Krasnova, O.A. Voeiko, Generative design as a new stage of design, In the collection: Quality management at the stages of the life cycle of technical and technological systems. collection of scientific papers of the All-Russian Scientific and Technical Conference. Southwest State University, 125-129 (2019)

13. D.O. Fedchun, R.E. Tlusty, Architecture and design: history, theory, innovations 2, 238241 (2017)

14. P. Janssen, K.W. Chen, A. Mohanty, Automated Generation of BIM Models, Proceedings of the 34th eCAADe Conference, Newcastle, UK, 583-590.

15. P.S. Zhalnina, T.P. Bilyushova, Architecture and design: history, theory, innovations 3, 31-37 (2018) 